\title{
Noncoding mutations target cis-regulatory elements of the FOXA1 plexus in prostate cancer
}

Stanley Zhou (10 1,2, James R. Hawley (10 1,2, Fraser Soares (1) 1, Giacomo Grillo1, Mona Teng 1,2, Seyed Ali Madani Tonekaboni², Junjie Tony Hua,2, Ken J. Kron, Parisa Mazrooei,2, Musaddeque Ahmed, Christopher Arlidge ${ }^{1}$, Hwa Young Yun ${ }^{1}$, Julie Livingstone (10 ${ }^{3}$, Vincent Huang (10 ${ }^{3}$, Takafumi N. Yamaguchi (10 ${ }^{3}$, Shadrielle M.G. Espiritu, Yanyun Zhu4, Tesa M. Severson ${ }^{4}$, Alex Murison', Sarina Cameron', Wilbert Zwart ${ }^{4,5}$, Theodorus van der Kwast (10 6, Trevor J. Pugh (10) 1,2,3, Michael Fraser ${ }^{3}$, Paul C. Boutros (1) 2,3,7,8,9,10,11, Robert G. Bristow (1) 1,2,12,13,14,15, Housheng Hansen $\mathrm{He}^{1,2}$ \& Mathieu Lupien (1) 1,2,3*

Prostate cancer is the second most commonly diagnosed malignancy among men worldwide. Recurrently mutated in primary and metastatic prostate tumors, FOXA1 encodes a pioneer transcription factor involved in disease onset and progression through both androgen receptor-dependent and androgen receptor-independent mechanisms. Despite its oncogenic properties however, the regulation of FOXA1 expression remains unknown. Here, we identify a set of six cis-regulatory elements in the FOXA1 regulatory plexus harboring somatic singlenucleotide variants in primary prostate tumors. We find that deletion and repression of these cis-regulatory elements significantly decreases FOXA1 expression and prostate cancer cell growth. Six of the ten single-nucleotide variants mapping to FOXA1 regulatory plexus significantly alter the transactivation potential of cis-regulatory elements by modulating the binding of transcription factors. Collectively, our results identify cis-regulatory elements within the FOXA1 plexus mutated in primary prostate tumors as potential targets for therapeutic intervention.

\footnotetext{
${ }_{1}^{1}$ Princess Margaret Cancer Centre, University Health Network, Toronto, ON, Canada. ${ }^{2}$ Department of Medical Biophysics, University of Toronto, Toronto, ON, Canada. ${ }^{3}$ Ontario Institute for Cancer Research, Toronto, ON, Canada. ${ }^{4}$ Division of Oncogenomics, Oncode Institute, the Netherlands Cancer Institute, Amsterdam, The Netherlands. ${ }^{5}$ Laboratory of Chemical Biology and Institute for Complex Molecular Systems, Department of Biomedical Engineering, Eindhoven University of Technology, Eindhoven, The Netherlands. ${ }^{6}$ Department of Laboratory Medicine and Pathobiology, University of Toronto, Toronto, ON, Canada. ${ }^{7}$ Department of Pharmacology and Toxicology, University of Toronto, Toronto, CA, Canada. ${ }^{8}$ Department of Human Genetics, University of California, Los Angeles, CA, USA. ${ }^{9}$ Department of Urology, University of California, Los Angeles, CA, USA. ${ }^{10}$ Institute for Precision Health, University of California, Los Angeles, CA, USA. ${ }^{11}$ Jonsson Comprehensive Cancer Center, University of California, Los Angeles, CA, USA. ${ }^{12}$ Department of Radiation Oncology, University of Toronto, Toronto, ON, Canada. ${ }^{13}$ CRUK Manchester Institute and Manchester Cancer Research Centre, Manchester, UK. ${ }^{14}$ Division of Cancer Sciences, Faculty of Biology, Health and Medicine, University of Manchester, Manchester, UK. ${ }^{15}$ The Christie NHS Foundation Trust, Manchester, UK. *email: mlupien@uhnresearch.ca
} 
$\mathrm{P}$ rostate cancer is the second most commonly diagnosed cancer among men with an estimated 1.3 million new cases worldwide in $2018^{1}$. Although most men diagnosed with primary prostate cancer are treated with curative intent through surgery or radiation therapy, treatments fail in $30 \%$ of patients within 10 years $^{2}$ resulting in a metastatic disease ${ }^{3}$. Patients with metastatic disease are typically treated with anti-androgen therapies, the staple of aggressive prostate cancer treatment ${ }^{4}$. Despite the efficacy of these therapies, recurrence ultimately develops into lethal metastatic castration resistant prostate cancer (mCRPC) ${ }^{4}$. As such, there remains a need to improve our biological understanding of prostate cancer development and find novel strategies to treat patients.

Sequencing efforts identified coding somatic single-nucleotide variants (SNVs) mapping to FOXA1 in up to $9 \%{ }^{5-10}$ and $13 \%{ }^{9-11}$ of primary and mCRPC patients, respectively. These coding somatic SNVs target the Forkhead and transactivation domains of FOXA $1^{12}$, altering its pioneering functions to promote prostate cancer development ${ }^{10,13}$. Outside of coding SNVs, whole-genome sequencing also identified somatic SNVs and indels in the 3'UTR and C-terminus of FOXA1 in $\sim 12 \%$ of mCPRC patients ${ }^{14}$. In addition to SNVs, the FOXA1 locus is a target of structural rearrangements in both primary and metastatic prostate cancer tumors, inclusive of duplications, amplifications, and translocations ${ }^{9,10}$. Taken together, FOXA1 is recurrently mutated taking into account both its coding and flanking noncoding sequences across various stages of prostate cancer development.

FOXA1 serves as a pioneer transcription factor (TF) that can bind to heterochromatin, promoting its remodeling to increase accessibility for the recruitment of other $\mathrm{TFs}^{15}$. FOXA1 binds to chromatin at cell-type specific genomic coordinates facilitated by the presence of mono- and dimethylated lysine 4 of histone $\mathrm{H} 3$ (H3K4me1 and $\mathrm{H} 3 \mathrm{~K} 4 \mathrm{me} 2$ ) histone modifications ${ }^{16,17}$. In prostate cancer, FOXA1 is known to pioneer and reprogram the binding of the androgen receptor (AR) alongside HOXB13 ${ }^{18}$. Independent from its role in AR signaling, FOXA1 also regulates the expression of genes involved in cell cycle regulation in prostate cancer $^{19-21}$. For instance, FOXA1 co-localizes with CREB1 to regulate the transcription of genes involved in cell cycle processes, nuclear division, and mitosis in mCRPC ${ }^{19-25}$. FOXA1 has also been shown to promote feed-forward mechanisms to drive disease progression ${ }^{26,27}$. Hence, FOXA1 contributes to ARdependent and AR-independent processes favouring prostate cancer development.

Despite the oncogenic roles of FOXA1, therapeutic avenues to inhibit its activity in prostate cancer are lacking. In the breast cancer setting for instance, the use of cyclin-dependent kinases inhibitors have been suggested based on their ability to block FOXA1 activity on chromatin ${ }^{28}$. As such, understanding the governance of FOXA1 mRNA expression offers an alternative strategy to find modulators of its activity. Gene expression relies on the interplay between distal cis-regulatory elements (CREs), such as enhancers and anchors of chromatin interaction, and their target gene promoter $(s)^{29}$. These elements can lie tens to hundreds of kilobases (kbp) away from each other on the linear genome but physically engage in close proximity with each other in the three-dimensional space $^{30}$. By measuring contact frequencies between loci through the use of chromatin conformation capture-based technologies, it enables the identification of regulatory plexuses corresponding to sets of CREs in contact with each other ${ }^{31,32}$. By leveraging these technologies, we can begin to understand the three-dimensional organization of the prostate cancer genome and delineate the FOXA1 regulatory plexus.

Here, we integrate epigenetics and genetics from prostate cancer patients and model systems to delineate CREs establishing the regulatory plexus of FOXA1. We functionally validate a set of six mutated CREs that regulate FOXA1 mRNA expression. We further show that SNVs mapping to these CREs are capable of altering their transactivation potential, likely through modulating the binding of key prostate cancer TFs.

\section{Results}

FOXA1 is essential for prostate cancer proliferation. We interrogated FOXA1 expression levels across cancer types. We find that FOXA1 mRNA is consistently the most abundant in prostate tumors compared with 25 other cancer types across patients (Fig. 1a), ranking in the 95th percentile for 492 of 497 prostate tumors profiled in TCGA (Supplementary Fig. 1a). Using the same data set we also find that FOXA1 is the most highly expressed out of 41 other Forkhead Box (FOX) factors in prostate tumors (Supplementary Fig. 1b). We next analyzed expression data from DEPMAP and observed FOXA1 to be most highly expressed in prostate cancer cell lines compared with cell lines of other cancer types (Supplementary Fig. 2a). Among the eight prostate cancer cell lines in the dataset (22Rv1, DU145, LNCaP, MDA-PCa-2B, NCI-H660, PrECLH, PC3, and VCaP), FOXA1 mRNA abundance is above the 90th percentile in all but one cell line $(\mathrm{PrECLH})$ compared with the $>56,000$ protein coding and non-protein coding genes profiled (Supplementary Fig. 2b). These new results gained from the TCGA and DEPMAP validate previous understanding that FOXA1 is one of the highest expressed genes in prostate cancer ${ }^{33}$

Following up on FOXA1 mRNA expression levels, we interrogated the essentiality of FOXA1 for prostate cancer cell growth. RNAi-mediated essentiality screens compiled in DEPMAP show that FOXA1 lies in the 94th percentile across six of the eight available prostate cancer cell lines: $22 \mathrm{Rv} 1, \mathrm{LNCaP}, \mathrm{MDA}$ PCa 2B, NCI-H660, PC3, and VCaP cells (Fig. 1b, c). The median RNAi-mediated essentiality score for all prostate cell lines is significantly lower than all other cell lines, suggesting that FOXA1 is especially essential for prostate cancer cell proliferation (permutation test, $p=1 \times 10^{-6}$, see Methods) (Supplementary Fig. 3a). Growth assays in $\mathrm{LNCaP}$ and $\mathrm{VCaP}$ cells following FOXA1 knockdown using two independent siRNAs (Fig. 1d, Supplementary Fig. 3b) show significant growth inhibition in LNCaP (siRNA \#1: fourfold, siRNA \#2: 3.35-fold) and $\mathrm{VCaP}$ (siRNA \#1: 8.7-fold, siRNA \#2: twofold) cells 5 days post transfection (Mann-Whitney $U$ test, $p<0.05$; Fig. 1e, f). In accordance with previous reports, our results using essentiality datasets followed by knockdown validation reveals that FOXA1 is oncogenic and essential for prostate cancer cell proliferation.

Identifying putative FOXA1 CREs. The interweaving of distal CREs with target gene promoters establishes regulatory plexuses with some to be ascribed to specific genes ${ }^{31,32}$. Regulatory plexuses stem from chromatin interactions orchestrated by various factors including ZNF143, YY1, CTCF, and the cohesin complex ${ }^{34-36}$. Motivated by the oncogenic role of FOXA1 in prostate cancer, we investigated its regulatory plexus controlling its expression. According to chromatin contact frequency maps generated from Hi-C assays performed in LNCaP prostate cancer cells, FOXA1 lies in a $440 \mathrm{kbp}$ TAD (chr14: 37720002-38160000 \pm 40 $\mathrm{kbp}$ adjusting for resolution) (Fig. 2a). By overlaying DNase-seq data from LNCaP prostate cancer cells, there are a total of 123 putative CREs reported as DNase I hypersensitive sites (DHS) that populate this TAD (Fig. 2a). We next inferred the regulatory plexus of FOXA1 using the cross cell-type correlation based on DNA accessibility (C3D) method ${ }^{37}$. C3D aggregates and draws correlation of DHS signal intensities between the cell line of choice and the DHS signal across all systems in the database $\mathrm{e}^{37}$. Anchoring our analysis to the FOXA1 promoter and using 

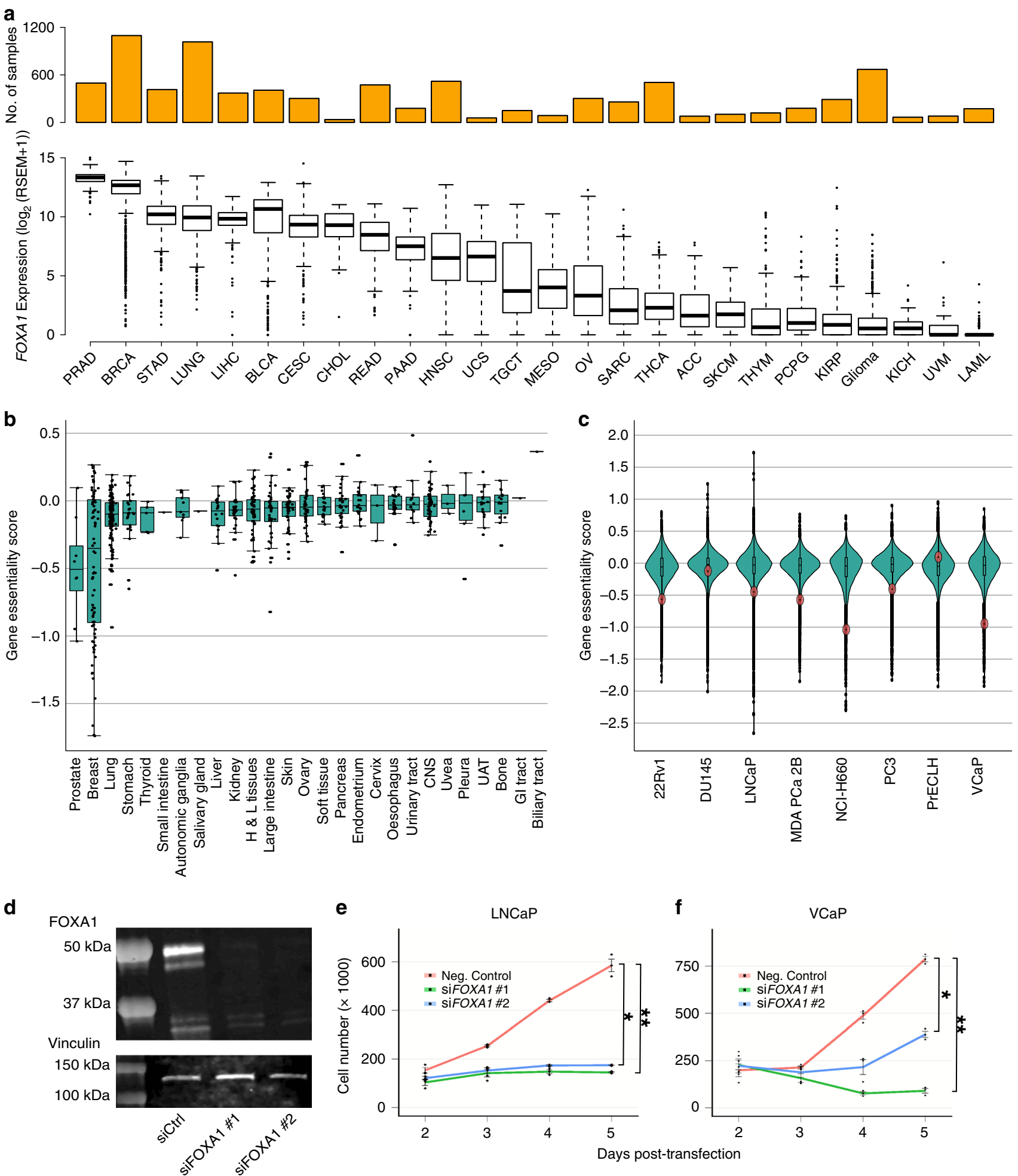

Fig. 1 FOXA1 is highly expressed in prostate cancer and essential for prostate cancer cell proliferation. a The mRNA expression of FOXA1 across tumor types $(n=26)$ from RNA-seq data of TCGA. b FOXA1 essentiality mediated through RNAi across various cell lines $(n=707)$ from DEPMAP. Gene essentiality scores are normalized $Z$ scores. Higher scores indicate less essential, and lower scores indicate more essential for cell proliferation. $X$ axis indicate tissue of origin for each cell line tested. Each dot indicates one cell line. c Gene essentiality mediated through RNAi across prostate cancer cell lines $(n=8)$ from DEPMAP. Each dot indicates one gene, red indicates FOXA1. d Representative western blot against FOXA1 in LNCaP cells 5 days post transfection of non-targeting siRNA and two independent siRNA targeting FOXA1. e Cell proliferation assay conducted in LNCaP cells upon siRNAmediated knockdown of FOXA1 across 5 days. $\mathbf{f}$ Cell proliferation assay conducted in VCaP cells upon siRNA-mediated knockdown of FOXA1 across 5 days. Error bars indicate \pm s.d. $n=3$ independent experiments. Mann-Whitney $U$ test, ${ }^{\star} p<0.05,{ }^{\star \star} p<0.01$. 
a

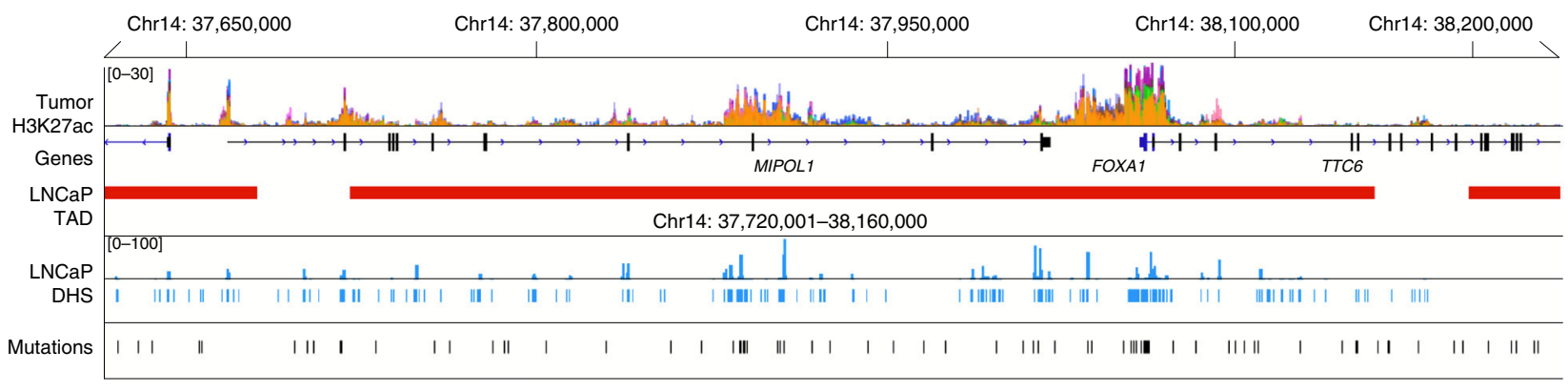

b

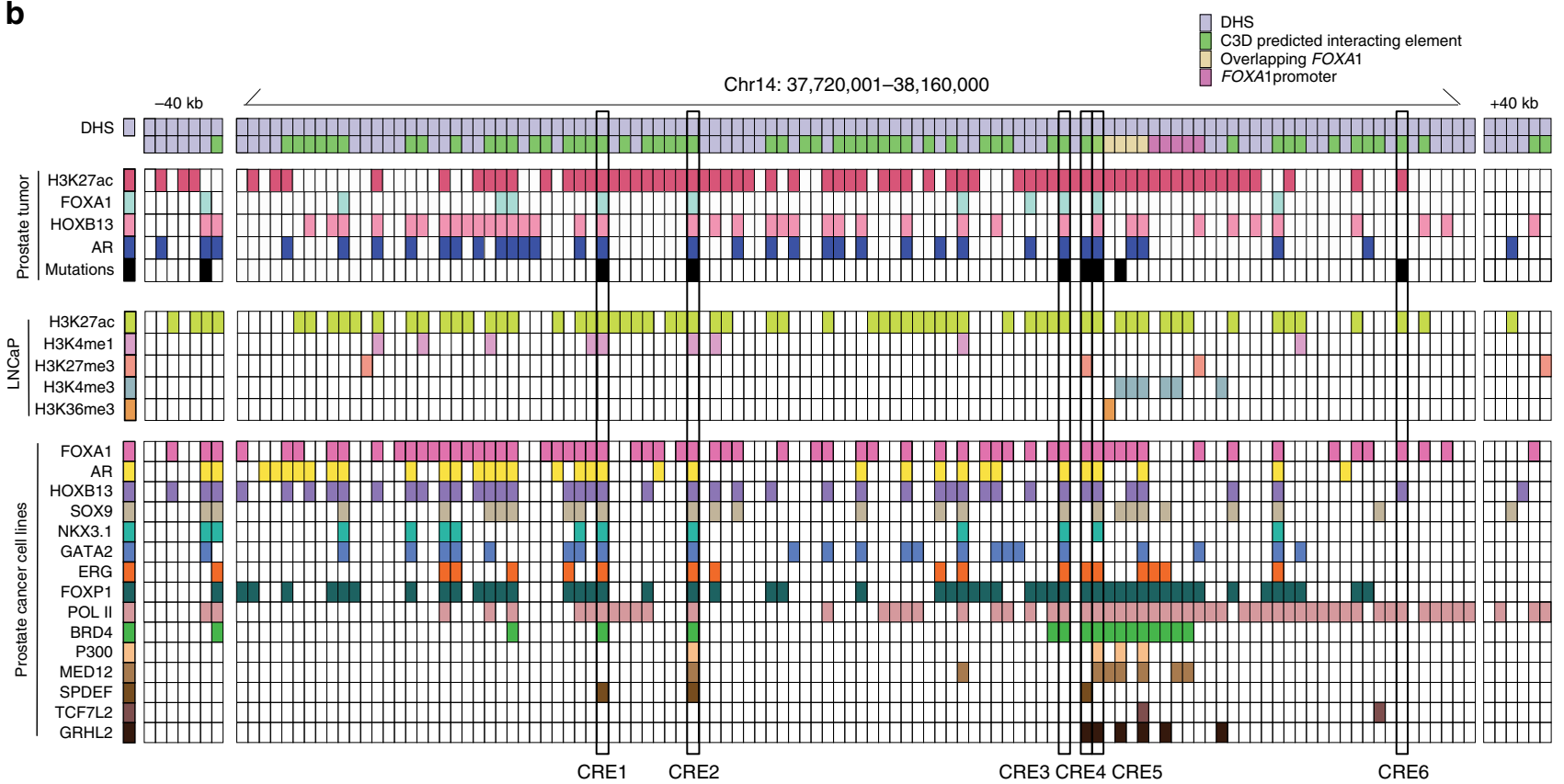

Fig. 2 Epigenetic annotation of 14q21.1 locus and identification of FOXA1 CREs. a Overview of cis-regulatory landscape surrounding FOXA1 on the $14 q 21.1$ locus. H3K27ac signal track is the ChIP-seq signal overlay of 19 primary prostate tumors ${ }^{38}$. LNCaP Hi-C depicts the TAD structure around FOXA1. Mutations indicate SNVs identified in 200 primary prostate tumors ${ }^{6,40}$. b Functional annotation of putative FOXA1 CREs using transcription factor and histone modification ChIP-seq conducted in primary tumors and prostate cancer cell lines. Annotated in the matrix are all DHS within the TAD and \pm 40 $\mathrm{kbp}$ resolution left and right of the TAD. Putative FOXA1 CREs targeted by noncoding SNVs for downstream validation are boxed.

accessible chromatin regions defined in $\mathrm{LNCaP}$ prostate cancer cells identified 55 putative CREs to the FOXA1 regulatory plexus $(r>0.7)$ (Fig. 2b).

Putativhe FOXA1 CREs harbor TF-binding sites and SNVs. To delineate the CREs that could be actively involved in the transcriptional regulation of FOXA1, we annotated the DHS with available ChIP-seq data for histone modifications and TFs conducted in LNCaP, 22Rv1, VCaP prostate cancer cell lines and primary prostate tumors (Fig. 2b) ${ }^{18,38}$. Close to $60 \%(33 / 55)$ of the putative FOXA1 plexus CREs are positively marked by $\mathrm{H} 3 \mathrm{~K} 27 \mathrm{ac}$ profiled in primary prostate tumors ${ }^{38}$, indicative of active CREs in tumors (Fig. 2b) ${ }^{39}$. Next, considering that noncoding SNVs can target a set of CREs that converge on the same target gene in cancer ${ }^{32}$, we overlapped the somatic SNVs called from the whole-genome sequencing across 200 primary prostate tumors to the $33 \mathrm{H} 3 \mathrm{~K} 27 \mathrm{ac}$-marked DHS predicted to regulate FOXA1 (Supplementary Data) ${ }^{6,40}$. This analysis identified 6 out of the 33 DHS marked with H3K27ac (18.2\%) harboring one or more SNV(s) (10 total SNVs called from nine tumors) (Fig. 2b). We observe that these six CREs can be bound by multiple TFs in prostate cancer cells, including FOXA1, AR, and HOXB13 (Fig. 2b, Supplementary Fig. 4a-f). The Hi-C data from the
LNCaP prostate cancer cells corroborates the C3D predictions as demonstrated by the elevated contact frequency between the region harboring the FOXA1 promoter and where the six CREs are located, compared with other loci in the same TAD (Fig. 3a). The six CREs lie in intergenic or intronic regions (Fig. $3 \mathrm{~b}-\mathrm{h}$ ). Together, histone modifications, TF-binding sites and noncoding SNVs support that these six putative CREs are active in primary prostate cancer. The $\mathrm{Hi}-\mathrm{C}$ and $\mathrm{C} 3 \mathrm{D}$ predictions suggest that they regulate FOXA1 expression.

Disruption of CREs reduces FOXA1 mRNA expression. We next assessed the role of CREs toward FOXA1 expression using LNCaP and 22Rv1 clones stably expressing the wild-type Cas9 protein (Fig. 4a, b). Guide RNAs (gRNAs) designed against the FOXA1 gene (exon 1 and intron 1 ) served as positive control while an outside-TAD region (i.e., termed Chr14 (-)), a region on a different chromosome (the human AAVS1 safe-harbor site at the PPP1R12C locus ${ }^{38,41}$ ), and three regions within the TAD predicted to be excluded from the FOXA1 plexus served as negative controls (Supplementary Data). Individual deletion of the FOXA1 plexus CREs through transient transfection of gRNAs into the LNCaP cells (See Methods) led to significantly decreased FOXA1 mRNA expression ( $\triangle \mathrm{CRE} 1 \sim 29.3 \pm 8.3 \%$, 

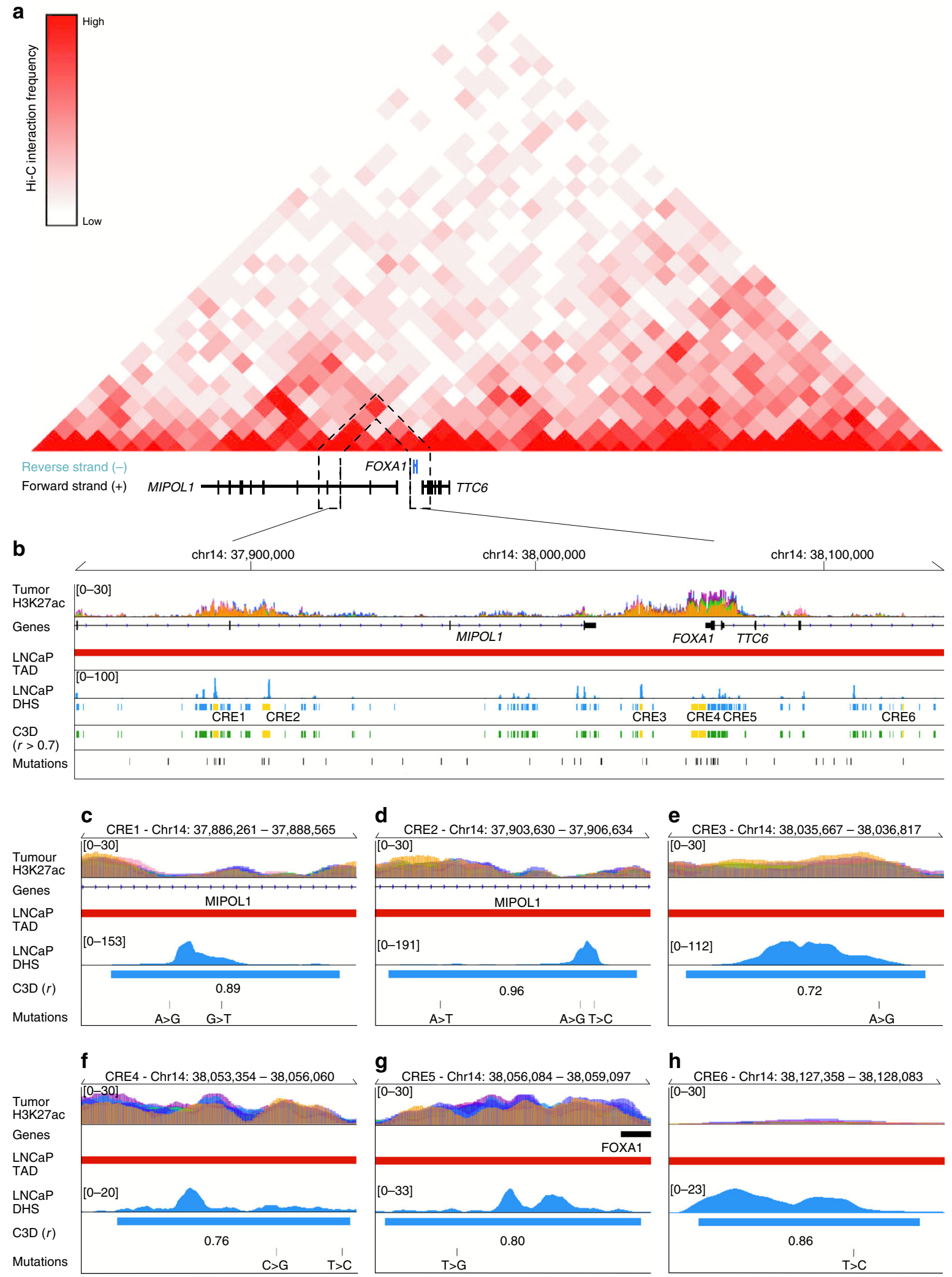

Fig. 3 Putative CREs predicted to interact with FOXA1 promoter. a $\mathrm{Hi}-\mathrm{C}$ conducted in $\mathrm{LNCaP}$ cells indicating physical interactions between putative FOXA1 CREs and FOXA1 promoter. Hi-C resolution is $40 \mathrm{kbp}$. $\mathbf{b}$ The six putative FOXA1 CREs are colored in yellow. c-h Zoom-in of each individual putative FOXA1 CRE. C3D ${ }^{37}$ value is Pearson correlation of DHS signal between LNCaP and the DHS reference matrix. 
a

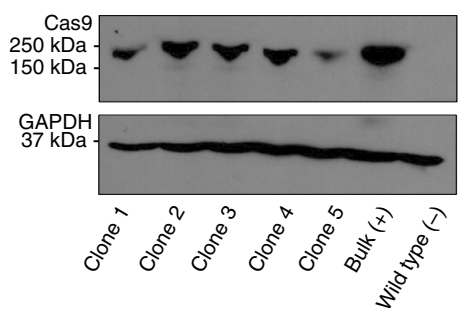

c

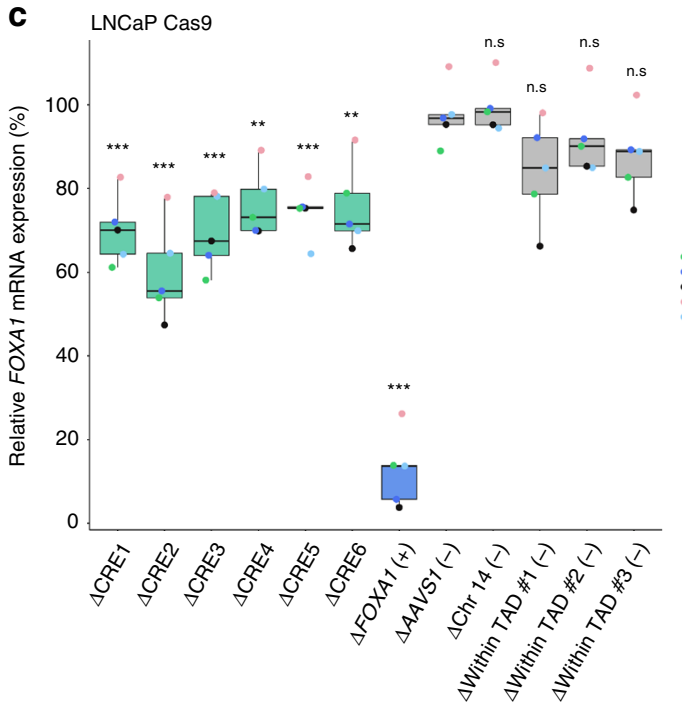

e
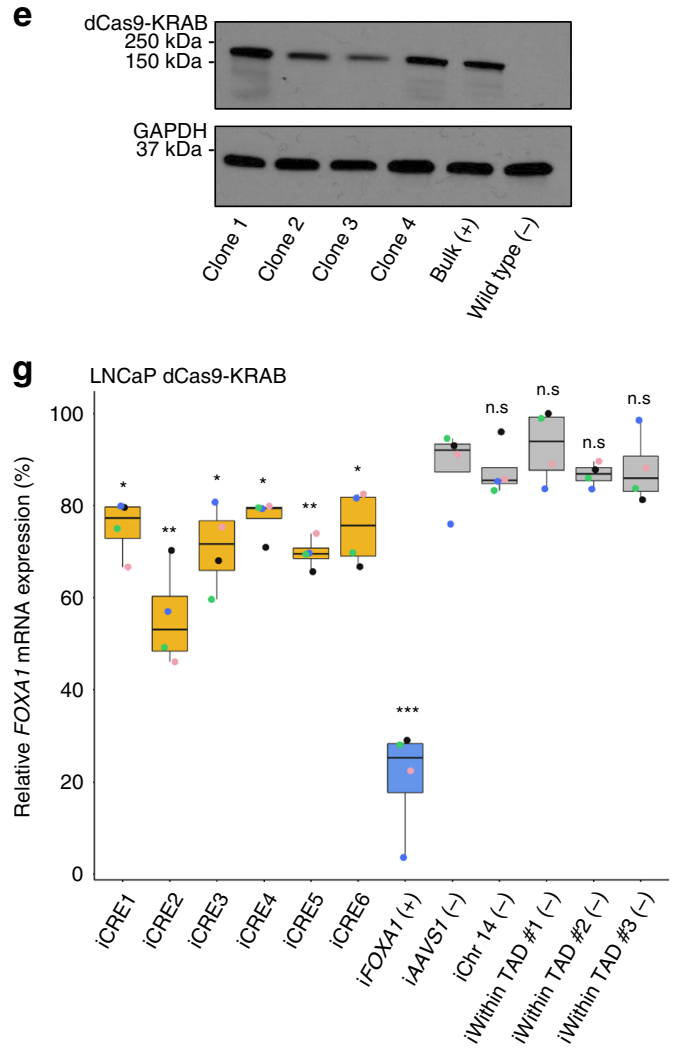

$\triangle$ CRE2 $\sim 40.1 \pm 11.8 \%, \triangle$ CRE $3 \sim 30.6 \pm 9.1 \%, \triangle$ CRE $4 \sim 23.6 \pm$ $8.2 \%, \Delta$ CRE $5 \sim 25.3 \pm 6.6 \%, \Delta$ CRE $6 \sim 24.5 \pm 10.2 \%$ and $\triangle$ FOXA 1 (exon 1 and intron 1) $\sim 87.4 \pm 8.8 \%$ reduction relative to basal levels) (Fig. 4c, Supplementary Fig. 5a-f). In contrast, deletion of several negative control regions within the same TAD did not b

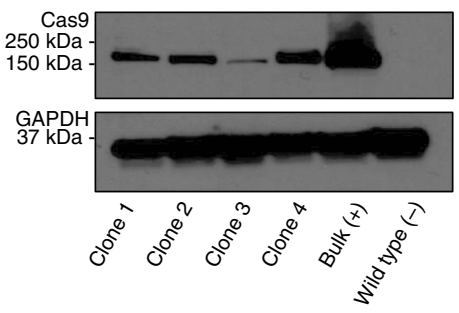

d

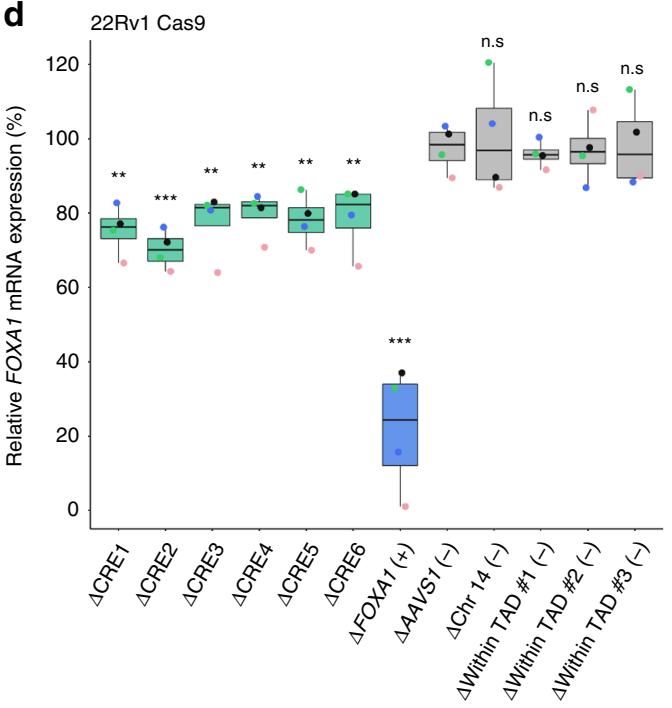

f

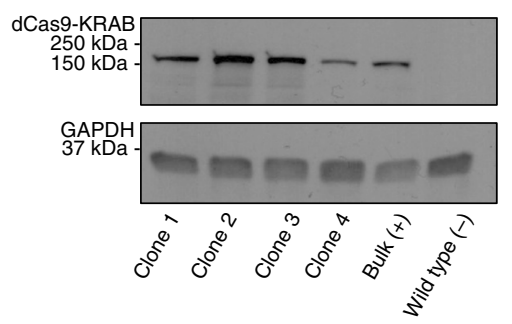

h

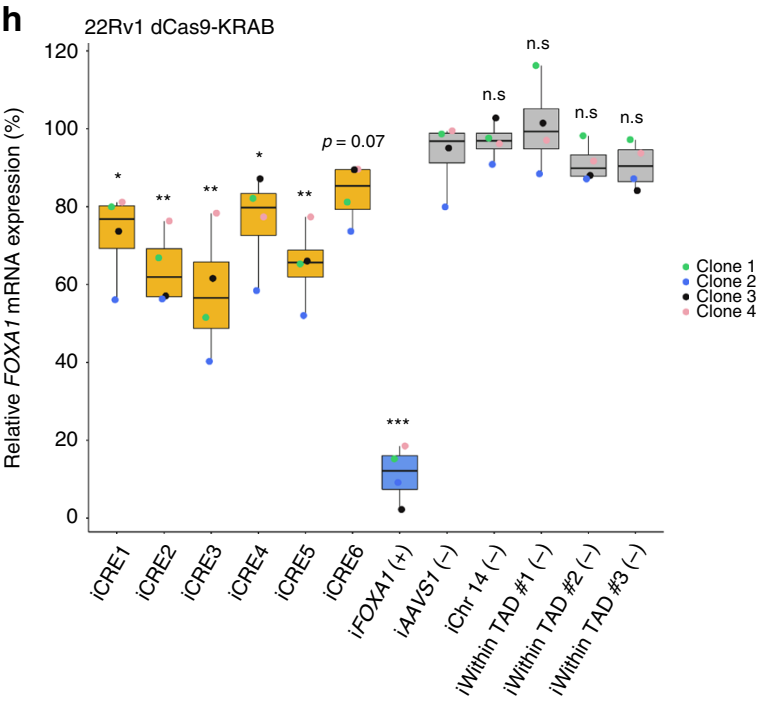

significantly reduce FOXA1 mRNA level (Fig. 4c, Supplementary Fig. $5 \mathrm{~g}-\mathrm{i})$. Similar results were observed in $22 \mathrm{Rv} 1$ prostate cancer cells (Fig. 4d). As each clone expressed Cas9 protein at different levels, there may be a difference between genome editing efficiencies between the clones. We compared the CRISPR/Cas9 
Fig. 4 Functional dissection of putative FOXA1 CREs. a Representative western blot probed against Cas9 in LNCaP clones ( $n=5$ independent clones) derived to stably express Cas9 protein upon blasticidin selection. b Representative western blot probed against Cas 9 in $22 \mathrm{Rv} 1$ clones ( $n=4$ independent clones) derived to stably express Cas9 protein upon blasticidin selection. c FOXA1 mRNA expression normalized to housekeeping TBP mRNA expression upon CRISPR/Cas9-mediated deletion of each CRE using LNCaP clones ( $n=5$ independent experiments, each dot represents an independent clone). d FOXA1 mRNA expression normalized to housekeeping TBP mRNA expression upon CRISPR/Cas9-mediated deletion of each CRE using 22Rv1 clones ( $n=4$ independent experiments, each dot represents an independent clone). e Representative western blot probed against Cas9 in LNCaP clones ( $n=4$ independent clones) derived to stably express the dCas9-KRAB fusion protein upon blasticidin selection. $\mathbf{f}$ Representative western blot probed against Cas9 in 22Rv1 clones ( $n=4$ independent clones) derived to stably express dCas9-KRAB fusion protein upon blasticidin selection. $\mathbf{g}$ FOXA1 mRNA expression normalized to housekeeping TBP mRNA expression upon dCas9-KRAB-mediated repression of each CRE using LNCaP clones ( $n=4$ independent experiments, each dot represents an independent clone). $\mathbf{h}$ FOXA1 mRNA expression normalized to housekeeping TBP mRNA expression upon dCas9-KRAB-mediated repression of each CRE using 22Rv1 clones ( $n=4$ independent experiments, each dot represents an independent clone). FOXA1 mRNA expression was normalized to basal FOXA1 expression prior to statistical testing. $\Delta$ indicates CRISPR/Cas9-mediated deletion, $\mathbf{i}$ indicates dCas9KRAB-mediated repression. Error bars indicate \pm s.d. Student's $t$ test, n.s not significant, ${ }^{\star} p<0.05,{ }^{\star \star} p<0.01,{ }^{\star \star \star} p<0.001$.

on-target genome editing efficiency across the five LNCaP cell line-derived clones with the relative FOXA1 mRNA levels, and indeed observe a significant inverse correlation across all CREs (Pearson's correlation $r=0.49, \quad p<0.005$ ) (Supplementary Fig. 6a) and agreeing trends for each individual CRE (Supplementary Fig. 6b).

Complementary to our findings using the wild-type CRISPR/ Cas9 system, we next generated four LNCaP and four 22Rv1 cell line-derived dCas9-KRAB fusion protein expressing clones (Fig. 4e, f). Transient transfection of the same gRNAs used in the wild-type Cas9 experiments, targeting the six FOXA1 plexus CREs (Supplementary Data) into our dCas9-KRAB LNCaP clones significantly decreased FOXA1 expression relative to basal levels (iCRE1 24.6 $\pm 6.2 \%$, iCRE2 42.2 $\pm 10.8 \%$, iCRE3 25.3 $\pm 9.2 \%$, iCRE $4 \sim 23.3 \pm 4.3 \%$, iCRE5 $~ 30.2 \pm 3.4 \%$, and iCRE6 $23.1 \pm 8.1 \%$ reduction). Similarly, gRNAs targeting the dCas9KRAB fusion protein to FOXA1 decreased its expression (iFOXA1 $\sim 81.6 \pm 11.8 \%$ reduction; Student's $t$ test, $p<0.05$, Fig. $4 \mathrm{~g}$ ). Analogous results were also observed in our four clonal 22Rv1 dCas9-KRAB cell lines (Student's $t$ test, $p<0.05$, Fig. $4 \mathrm{~h}$ ). Collectively, our results suggest that the six CREs control FOXA1 expression.

We further assessed the regulatory activity of the six FOXA1 plexus CREs by testing the consequent mRNA expression on other genes within the same TAD, namely MIPOL1 and TTC6. $\triangle$ CRE1 and $\triangle$ CRE2 significantly reduced MIPOL1 mRNA expression by $\sim 38.4 \pm 6.4 \%$ and $\sim 48.4 \pm 9 \%$, respectively, relative to basal levels, whereas deletion of the other four CREs did not result in any significant MIPOL1 expression changes (Student's $t$ test, $p<0.05$, Supplementary Fig. 7a). On the other hand, deletion of CREs each significantly reduced TTC6 mRNA expression relative to its basal levels ( $\triangle \mathrm{CRE} 1 \sim 52.9 \% \pm 6.4 \%$, $\Delta$ CRE2 $~ 66 \pm 11.3 \%, \Delta$ CRE3 $~ 55.5 \pm 12.8 \%, \Delta$ CRE4 $44.9 \pm$ $10.6 \%, \triangle$ CRE5 $43.1 \pm 11.9 \%$ and $\triangle$ CRE6 $52.2 \pm 7.3 \%$ reduction (Student's $t$ test, $p<0.05$, Supplementary Fig. 7b), in agreement with the fact that TTC6 shares its promoter with FOXA1 as both genes are transcribed on opposing strands (Supplementary Fig. 7c).

Reduction in FOXA1 mRNA expression resulting from the deletion of FOXA1 plexus CREs may also impact gene expression downstream of FOXA1. We assessed the mRNA expression of several FOXA1 target genes, namely SNAI2, ACPP, and GRIN3A. Deletion of CREs resulted in significant change in SNAI2 (upregulation; $\triangle$ CRE1 $\sim 190 \%, \triangle \mathrm{CRE} 2 \sim 162.8 \%, \triangle \mathrm{CRE} 3 \sim$ $147.5 \%, \triangle$ CRE4 133.3\%, $\triangle$ CRE5 137.3\%, $\triangle$ CRE6 120.8\%, $\triangle$ FOXA1 266.7\%), ACPP (downregulation; $\triangle$ CRE1 73.5\%, $\triangle$ CRE2 $\sim 62.5 \%, \triangle$ CRE3 $\sim 69.6 \%, \triangle$ CRE4 $\sim 75.6 \%, \Delta$ CRE5 70.9\%, $\triangle$ CRE6 $\sim 74.6 \%, \triangle F O X A 1 \sim 52.2 \%)$ and GRIN3A expression (upregulation; $\triangle \mathrm{CRE} 1 \sim 138.2 \%, \triangle \mathrm{CRE} 2 \sim 168.8 \%, \triangle \mathrm{CRE} 3$ $\sim 144.6 \%, \Delta$ CRE4 $~ 132.1 \%, \Delta$ CRE5 131.4\%, $\triangle$ CRE6 127\%, $\triangle F O X A 1 \sim 228 \%$ ) (Student's $t$ test, $p<0.05$, Supplementary Fig. $7 \mathrm{~d}-\mathrm{f})$. Collectively, our results support the regulation of most FOXA1 plexus CREs towards FOXA1 and its target genes.

FOXA1 CREs collaborate to regulate its expression. Expanding on the idea that multiple CREs can converge to regulate the expression of a single target gene $\mathrm{e}^{31,32,42}$, we asked whether the CREs we identified collaboratively regulate FOXA1 mRNA expression. Here, we applied a transient approach that delivers Cas9 protein:gRNA as a ribonucleoprotein (RNP) complex formed prior to transfection that would avoid the heterogeneity of Cas9 protein expression across the prostate cancer cell clones (See Methods). We first validated this system through single CRE deletions, where we transiently transfected a set of gRNA targeting the CRE of interest. In accordance with data from our prostate cancer cell clones stably expressing wild-type Cas9 and dCas9-KRAB, individual CRE deletion resulted in a significant reduction in FOXA1 mRNA expression: $\triangle \triangle \mathrm{CRE} 1 \sim 29.3 \pm 7.3 \%$, $\triangle \mathrm{CRE} 2 \sim 36 \pm 11.8 \%, \Delta \mathrm{CRE} 3 \sim 30.6 \pm 12.7 \%, \Delta \mathrm{CRE} 4 \sim 24.5 \pm$ $6.1 \%, \quad \Delta$ CRE5 $\sim 23.7 \pm 13.2 \%, \quad \Delta$ CRE6 $\sim 26.8 \pm 14.2 \%$ and $\triangle F O X A 1 \sim 96.2 \pm 1.4 \%$ reduction (Student's $t$ test, $p<0.05$, Fig. 5a, Supplementary Fig. 8a-f). Next for combinatorial deletions, we prioritized the CREs that harbor more than one SNV(i.e, CRE1, CRE2, CRE4), and transiently transfected RNP complexes that target both CREs in various combinations (i.e., CRE1 + CRE2, CRE1 + CRE4, CRE2 + CRE4), and assessed FOXA1 mRNA expression. Compared to negative control regions, the combinatorial deletion of $\triangle \mathrm{CRE} 1+\triangle \mathrm{CRE} 2, \triangle \mathrm{CRE} 1$ $+\triangle \mathrm{CRE} 4$, and $\triangle \mathrm{CRE} 2+\triangle \mathrm{CRE} 4$ resulted in a significant $\sim 48.5 \pm 4.5 \%, \sim 50.4 \pm 2.9 \%$, and $\sim 45.2 \pm 5.5 \%$ reduction in FOXA1 mRNA expression, respectively (Student's $t$ test, $p<0.05$, Fig. 5b, Supplementary Fig. 9a-f) a fold reduction greater than single CRE deletions (Student's $t$ test, Supplementary Fig. 10, $p<$ 0.05). These results together demonstrate that these CREs collaboratively contribute to the establishment and regulation of FOXA1 expression in prostate cancer.

Disruption of FOXA1 CREs reduces prostate cancer cell growth. As FOXA1 is essential for prostate cancer growth (Fig. 1b-e), we next sought to assess the importance of the six FOXA1 plexus CREs towards prostate cancer cell growth. We adapted a lentiviral-based approach that expressed both the Cas9 protein and two gRNA that target each CRE for deletion (See Methods). Upon lentiviral transduction with subsequent selection, we separated LNCaP prostate cancer cells for RNA, DNA, and for cell proliferation. We first tested the system by measuring FOXA1 mRNA expression, and independently observed significant reductions of FOXA1 mRNA expression $(\triangle \mathrm{CRE} 1 \sim 18 \%$, $\triangle \mathrm{CRE} 2 \sim 30 \%, \Delta \mathrm{CRE} 3 \sim 15 \%, \Delta \mathrm{CRE} 4 \sim 12 \%, \Delta \mathrm{CRE} 5 \sim 35 \%$, 

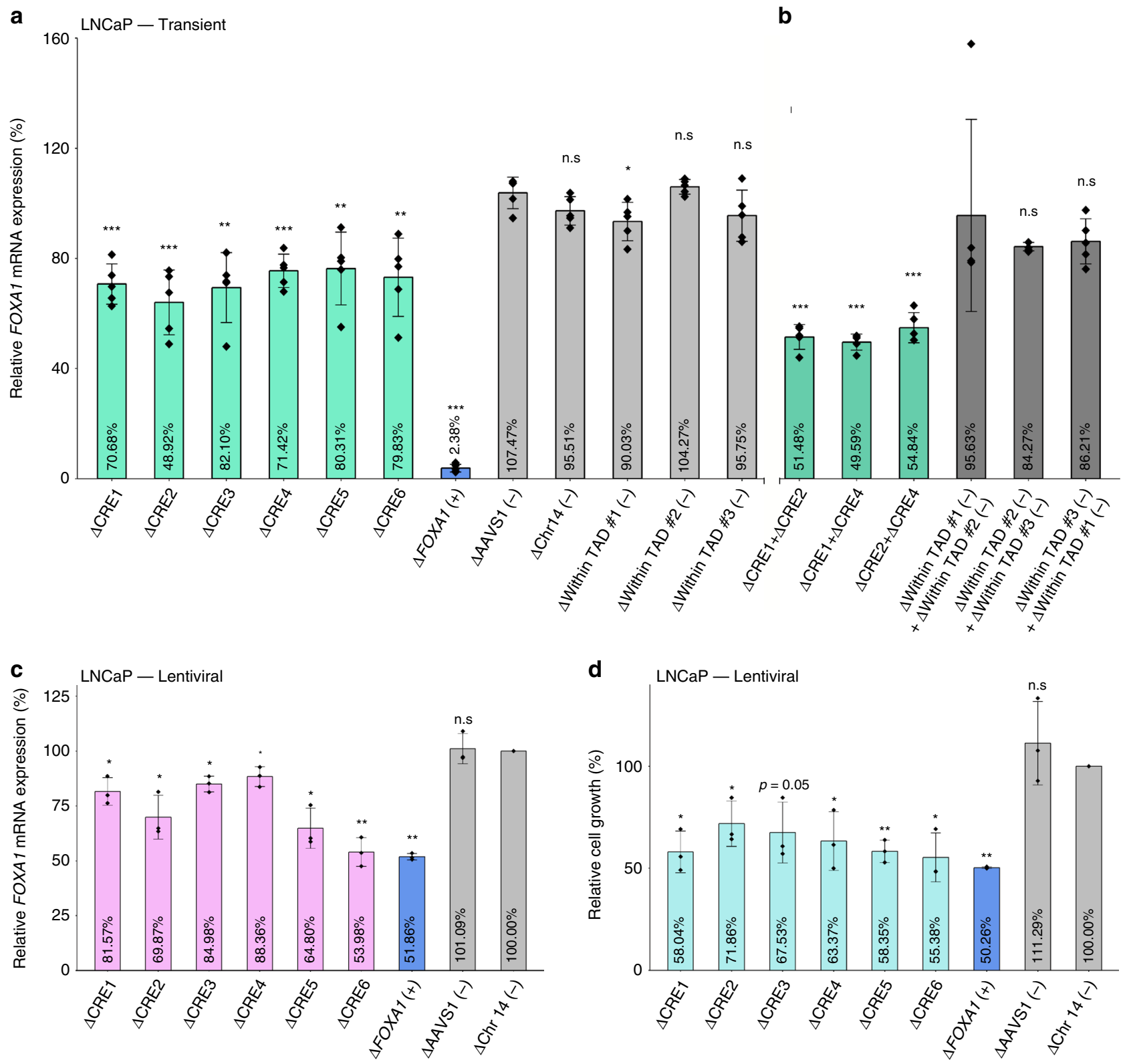

Fig. 5 FOXA1 CREs collaborate to regulate its expression and are critical for prostate cancer cell proliferation. a FOXA1 mRNA expression normalized to housekeeping TBP mRNA expression upon transient transfection-based CRISPR/Cas9-mediated deletion of CRE1, CRE2, CRE4, and sequential deletion combinations ( $n=5$ independent experiments). b FOXA1 mRNA expression normalized to housekeeping TBP mRNA expression upon bulk lentiviral-based CRISPR/Cas9-mediated deletion of each CRE in LNCaP cells ( $n=3$ independent experiments). c Cell proliferation assay conducted after puromycin and blasticidin selection for LNCaP cells carrying deleted region of interest. Data were based on cell counting 6 days after seeding post-selection ( $n=3$, representative of three independent experiments). FOXA1 mRNA expression upon deletion was normalized to basal FOXA1 expression prior to statistical testing. FOXA1 mRNA expression was normalized to basal LNCaP FOXA1 expression prior to statistical testing. $\Delta$ indicates CRISPR/Cas9-mediated deletion. Error bars indicate \pm s.d. Student's $t$ test, n.s not significant, ${ }^{\star} p<0.05,{ }^{\star \star} p<0.01,{ }^{\star \star \star} p<0.001$.

$\triangle$ CRE6 $\sim 46 \%$, and $\triangle F O X A 1$ (exon 1 and intron 1 ) $\sim 48 \%$ reduction (Student's $t$ test, $p<0.05$, Fig. $5 c$, Supplementary Fig. 11a-f). We then seeded these cells at equal density. Six days post seeding, we harvested the cells and observed a significant reduction in cell growth upon deleting any of the six FOXA1 plexus CREs ( $\triangle$ CRE1 $\sim 42 \%, \triangle \mathrm{CRE} 2 \sim 28 \%, \triangle \mathrm{CRE} 3 \sim 33 \%$, $\triangle$ CRE4 $\sim 27 \%, \triangle$ CRE5 $~ 42 \%, \triangle$ CRE6 $\sim 44 \%$ and $\triangle F O X A 1$ (exon 1 and intron 1) $\sim 50 \%$ reduction (Student's $t$ test, $p<0.05$, Fig. 5d). These results suggest that the six FOXA1 plexus contribute to prostate cancer etiology, in agreement with their ability to regulate FOXA1 expression and the essentiality of this gene in prostate cancer cell growth.
SNVs mapping to FOXA1 CREs can alter their activity. Singlenucleotide variants can alter the transactivation potential of $\mathrm{CREs}^{32,43-51}$. In total, we found 10 SNVs called from 9 out of the 200 tumors that map to the six FOXA1 plexus CREs (Fig. 6a, Supplementary Data). To assess the impact of these noncoding SNVs, we conducted luciferase assays comparing differential reporter activity between the variant and the wild-type allele of each CRE (Fig. 6b-k). We found that the variant alleles of 6 of the $10 \mathrm{SNV}$ s displayed significantly greater luciferase reporter activity when compared with the wild-type alleles (Mann-Whitney $U$ test, $p<0.05$ ). Specifically, we observed the following fold-changes: chr14:37,887,005 A $>$ G (1.65-fold), 

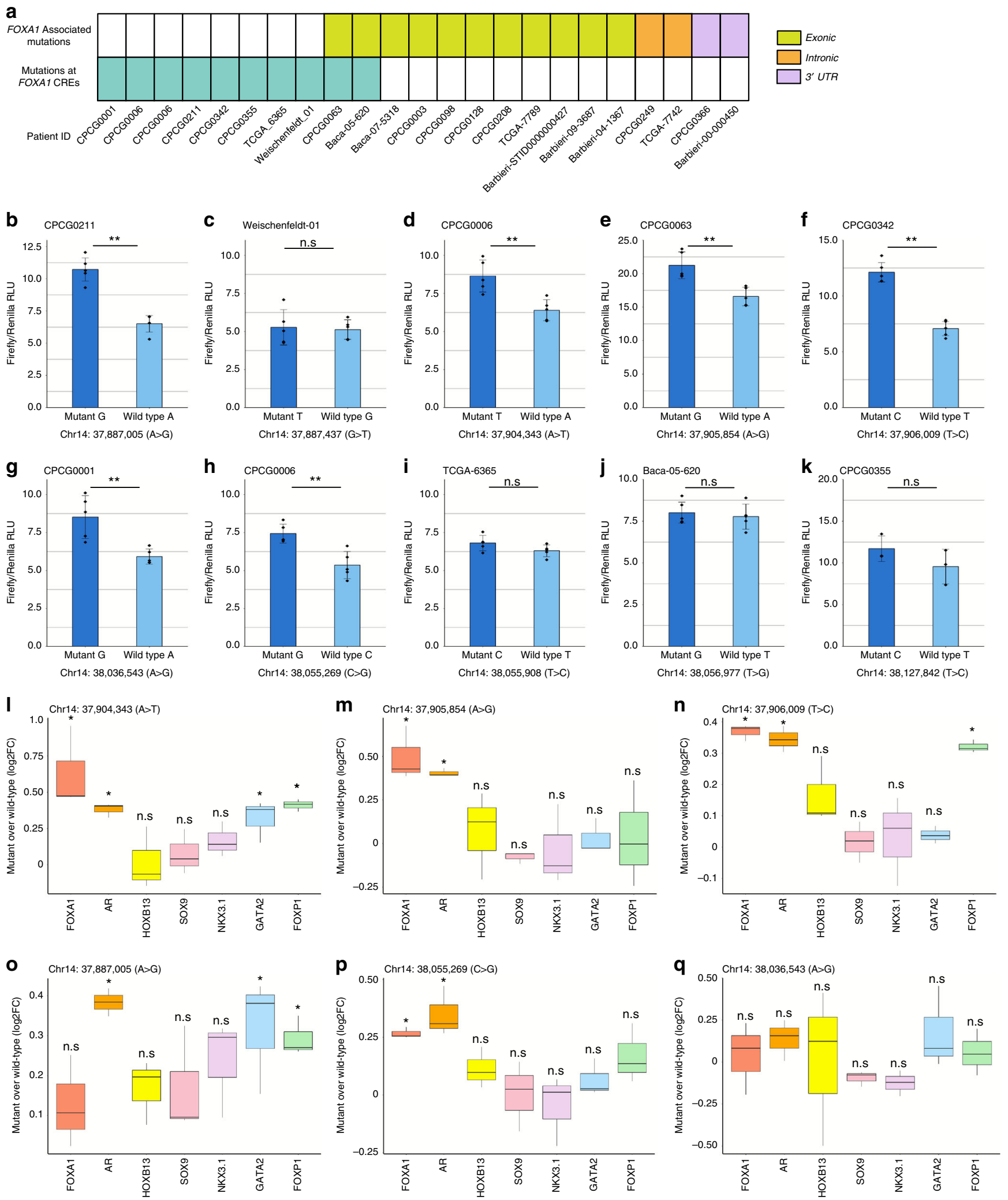

Fig. 6 Subset of noncoding SNVs mapping to the FOXA1 CREs are gain-of-function. a Matrix showcasing the patients from the Fraser et al. data set ${ }^{6}$ that harbor SNVs at the FOXA1 CREs, exons, introns, and the 3'UTR of FOXA1. b-k Luciferase assays conducted in LNCaP cells. Barplot showcases the mean firefly luciferase activity normalized by renilla luciferase activity, error bars indicate \pm s.d. $n=5$ independent experiments $(n=3$ for chr14: 38,127,842 (T > C)), and each diamond represents an independent experiment. Mann-Whitney $U$ test ${ }^{\star} p<0.05,{ }^{\star \star} p<0.01$. RLU = Relative Luciferase Unit. I-q Allelespecific ChIP-qPCR conducted on plasmids carrying the wild-type or variant sequence upon transient transfection in prostate cancer cells. Data is presented as $\log _{2}$ fold-change of variant sequence upon comparing to wild-type sequence ( $n=3$ independent experiments per ChIP). Student's $t$ test, n.s not significant, ${ }^{\star} p<0.05,{ }^{\star \star} p<0.01,{ }^{\star \star \star} p<0.001$. 
chr14:37,904,343 A> T (1.35-fold), chr14:37,905,854 A > G (1.28-fold), chr14:37,906,009 T > C (1.71-fold), chr14:38,036,543 A > G (1.44-fold), chr14:38,055,269 C > G (1.39-fold) (Fig. 6b-h). These results indicate that these SNVs can alter the transactivation potential of FOXA1 plexus CREs in prostate cancer cells.

SNVs mapping to FOXA1 CREs can modulate the binding of TFs. We next assessed if the changes in transactivation potential induced by noncoding SNVs related to changes in $\mathrm{TF}$ binding to CREs by allele-specific ChIP-qPCR ${ }^{32,44,51}$ in $\mathrm{LNCaP}$ prostate cancer cells. We observed differential binding of FOXA1, AR, HOXB13, GATA2, and FOXP1 for the chr14:37887005 $(\mathrm{A}>\mathrm{G})$ SNV found in CRE1; the chr14:37904343 $(\mathrm{A}>\mathrm{T})$, chr14:37905854 (A>G), and chr14:37906009 (T $>\mathrm{C})$ SNVs found in CRE2; and the chr14:38055269 (C> G) SNV found in CRE4 (Student's $t$ test, $p<0.05$, Fig. 6l-p). In contrast, SOX9 and NKX3.1 binding was unaffected by these SNVs (Fig. 6l-q). Compared with the wild-type sequence, chr14:37,887,005 A > G significantly increased AR binding (1.31-fold increase), GATA2 binding (1.25-fold increase) and FOXP1 binding (1.23-fold increase); chr14:37,904,343 $\mathrm{A}>\mathrm{T}$ significant increased $\mathrm{AR}$ binding (1.30-fold increase), GATA2 (1.25-fold increase) and FOXP1 (1.33-fold increase); chr14:37,905,854 A > G significantly increased FOXA1 binding (1.41-fold increase) and AR binding (1.33-fold increase); chr14:37,906,009 $\mathrm{T}>\mathrm{C}$ significantly increased the binding of FOXA1 (1.29-fold increase), AR (1.31fold increase), HOXB13 (1.13-fold increase) and FOXP1 (1.25fold increase); and chr14:38,055,269 C > G significantly increased FOXA1 binding (1.20-fold increase). Notably, all six SNVs increased the binding of the TFs known to bind at these CREs. In contrast, none of the SNVs significantly decreased the binding of these TFs. Our observations suggest that gain-of-function SNVs populate the FOXA1 plexus CREs.

\section{Discussion}

Modern technologies and understanding of the epigenome allow the possibility of probing $\mathrm{CRE}(\mathrm{s})$ involved in regulating genes implicated in disease. Despite FOXA1 being recurrently mutated ${ }^{5-8,11}$ and playing potent oncogenic roles in prostate cancer etiology $9,10,13$, the CREs involved in its transcriptional regulation are poorly understood. Understanding how FOXA1 is expressed can provide a complementary strategy to antagonize FOXA1 in prostate cancer.

We used the DHS profiled in prostate cancer cells to identify putative FOXA1 CREs by annotating these regions with five different histone modifications, TF-binding sites and noncoding SNVs profiled in prostate cancer cells and primary prostate tumors. Our efforts identified and validated a set of six active CREs involved in FOXA1 regulation, agreeing with a recent report where a subset of our CREs map to loci suggested to be in contact with the FOXA1 promoter $^{52}$. The disruption of these six distal CREs each significantly reduced FOXA1 mRNA levels, similar to what has been demonstrated for ESR1 in luminal breast cancer $^{32}$, MLH1 in Lynch syndrome ${ }^{53}$, MYC in lung adenocarcinoma and endometrial cancer ${ }^{54}$ and $A R$ in mCRPC $^{55,56 .}$ Through combinatorial deletion of two CREs, FOXA1 mRNA levels were further reduced in comparison with single CRE deletions, raising the possibility of CRE additivity ${ }^{57}$. The deletion of the FOXA1 plexus CREs also significantly reduced prostate cancer cell proliferation at levels comparable to what has been reported upon deletion of the amplified CRE upstream of the $A R$ gene in $\mathrm{mCRPC}^{55}$, suggestive of onco-CREs as reported in lung ${ }^{54}$, and prostate ${ }^{55}$ cancer.

More than $90 \%$ of SNVs found in cancer map to the noncoding genome ${ }^{58,59}$ with a portion of these SNVs mapping to CREs altering their transactivation potential ${ }^{32,44-46}$ and/or downstream target gene expression ${ }^{48,58,60}$. We extended this concept with SNVs identified from primary prostate tumors mapping to FOXA1 plexus CREs. We observed that a subset of these SNVs can alter transactivation potential by modulating the binding of specific TFs whose cistromes are preferentially burdened by SNVs in primary prostate cancer ${ }^{59}$. Our findings complement recent reports of SNVs found in the noncoding space of FOXA1 that could affect its expression ${ }^{14,61}$. The FOXA1 plexus CREs we identified here are also reported to be target of structural variants in both the primary and metastatic settings 9,62 , including tandem duplication in $\sim 14 \%(14 / 101)$ mCRPC tumors over CRE2 ${ }^{62}$, amplification, duplication, and translocation over CRE3, 4, 59. Notably, the translocation and duplication defining the FOXMIND enhancer driving FOXA1 expression reported in primary and metastatic settings harbors the CRE3 element we characterized $^{9}$. Collectively, these studies combined with our discoveries reveal the fundamental contribution of the FOXA1 plexus in prostate cancer etiology. As a whole, our findings in conjunction with recent reports suggest that CREs involved in the transcriptional regulation of FOXA1 may be hijacked in prostate tumors through various types of genetic alterations.

Despite initial treatment responses from treating aggressive primary and metastatic prostate cancer through castration to suppress AR signaling 4 , resistance ensues as $80 \%$ of mCRPC tumors harbor either $A R$ gene amplification, amplification of a CRE upstream of $A R$, or activating $A R$ coding mutations ${ }^{11,55,62}$. Given the AR-dependent ${ }^{15,18}$ and AR-independent ${ }^{25}$ oncogenic activity of FOXA1 in prostate cancer, its inhibition is an appealing alternative therapeutic strategy. Our dissection of the FOXA1 cis-regulatory landscape complement recent findings by revealing loci that are important for the regulation of FOXA1. Theoretically, direct targeting of the CREs regulating FOXA1 would downregulate FOXA1 levels and could therefore serve as a valid alternative to antagonize its function.

Taken together, we identified FOXA1 CREs targeted by SNVs that are capable of altering transactivation potential through the modulation of key prostate cancer TFs. The study supports the importance of considering CREs not only as lone occurrences but as a team that work together to regulate their target genes, particularly when considering the impact of genetic alterations. As such, our work builds a bridge between the understanding of FOXA1 transcriptional regulation and new routes to FOXA1 inhibition. Aligning with recent reports $9,10,13$, our findings support the oncogenic nature of FOXA1 in prostate cancer. Gaining insight on the cis-regulatory plexuses of important genes such as FOXA1 in prostate cancer may provide new avenues to inhibit other drivers across various cancer types to halt disease progression.

\section{Methods}

Cell culture. LNCaP and 22Rv1 cells were cultured in RPMI medium, and VCaP cells were cultured in DMEM medium, both supplemented with $10 \%$ FBS, and $1 \%$ penicillin-streptomycin at $37^{\circ} \mathrm{C}$ in a humidified incubator with $5 \% \mathrm{CO}_{2}$. These prostate cancer cells originated from ATCC. 293FT cells were purchased from ThermoFisher Scientific (Cat. No. R70007) maintained in complete DMEM medium (DMEM with 10\% FBS (080150, Wisent), L-glutamine (25030-081, ThermoFisher) and non-essential amino acids (11140-050, ThermoFisher) supplemented with $50 \mathrm{mg} / \mathrm{mL}$ Geneticin (4727894001, Sigma-Aldrich). The cells are regularly tested for Mycoplasma contamination. The authenticity of these cells was confirmed through short tandem repeat profiling.

\section{Prostate tumors and cancer cell lines expression. Cancer cell line mRNA} abundance data were collected from the Cancer Dependency Map Project (DEPMAP; https://depmap.org/portal/; RNA-seq TPM values from 2018q4 version) ${ }^{63}$ projects. Prostate tumor mRNA abundance data were collected from The Cancer Genome Atlas (TCGA) prostate cancer (TCGA-PRAD) project via the Xena 
Browser (https://xenabrowser.net/; data set description: TCGA prostate adenocarcinoma (PRAD) gene expression by RNA-seq (polyA+Illumina HiSeq; RSEM)).

Prostate cancer cell line gene essentiality. Essentiality scores were collected from the Cancer Dependency Map Project (DEPMAP) (https://depmap.org/portal/ download/; dataset description: $2018 \mathrm{q} 4$ versions of the "cell line metadata" and "combined RNAi"64, and all five non-cancer cell lines were removed (cell lines where the "Primary Disease" was listed in the metadata as one of the following: fibroblast, immortalized, immortalized_epithelial, non-cancerous, primary cells, or unknown). To compare gene essentiality between prostate cancer cell lines and others, essentiality scores for FOXA1 were collected from all available cell lines $(n=707)$. To perform a permutation test, the median of eight randomly selected cell lines was calculated one million times to generate a background distribution of essentiality scores across all cell types available. The median essentiality score from the eight prostate cancer cell lines was calculated and its percentile within the background distribution is reported.

siRNA knockdown and cell proliferation assay. In all, 300,000 LNCaP cells (Day 0) were reverse transfected with siRNA (siFOXA1 using Lipofectamine RNAimax reagent (ThermoFisher Scientific, Cat. No. 13778150)). Cells were counted using Countess automated cell counter (Invitrogen). Whole-cell lysates LNCaP cells after siRNA-mediated FOXA1 knockdown were collected at $96 \mathrm{~h}$ post transfection in RIPA buffer. Protein concentrations were determined through the bicinchoninic acid method (ThermoFisher Scientific, Cat. No. 23225). Then $25 \mu \mathrm{g}$ of lysate was subjected to SDS-PAGE. Upon completion of SDS-PAGE, protein was transferred onto PVDF membrane (Bio-Rad, Cat. No. 1704156). The membrane was blocked with $5 \%$ non-fat milk for $1 \mathrm{~h}$ at room temperature with shaking. After blocking, anti-FOXA1 (Abcam Cat. No. 23737) in 2.5\% non-fat milk was added, and was incubated at $4{ }^{\circ} \mathrm{C}$ overnight. Next day, the blot was washed and incubated with IRDye $800 \mathrm{CW}$ Goat Anti-Rabbit IgG secondary antibody (LI-COR, Cat. No. 925-32211) at room temperature for $1 \mathrm{~h}$. The blot was then washed and assessed with the Odyssey CLX imaging system (LI-COR).

Identifying putative FOXA1 CREs. Putative FOXA1 CREs were identified through the use of Cross Cell-Type Correlation based on DNase I Hypersensitivity (C3D) (https://github.com/tahmidmehdi/C3D) ${ }^{37}$. Predicted interacting DNase I Hypersensitivity Sites (DHS) with a Pearson's correlation above $0.7^{65}$ were kept for downstream analysis.

Hi-C and TADs in LNCaP cells. Hi-C and TADs conducted and called, respectively, in LNCaP cells is publicly available off ENCODE portal (ENCSR346DCU). Visualization of the Hi-C dataset is available on the $\mathrm{Hi}-\mathrm{C}$ Browser (http://promoter.bx.psu.edu/hi-c/) ${ }^{66}$.

Clonal wild-type Cas9 and dCas9-KRAB-mediated validation. Lentiviral particles were generated in 293FT cells (ThermoFisher) using the pMDG.2 and psPAX2 packaging plasmids (Addgene; \#12259 and \#12260, a gift from Didier Trono) alongside the Lenti-Cas9-2A-Blast plasmid (Addgene \#73310, gift from Jason Moffat) and collected $72 \mathrm{hrs}$ post transfection. LNCaP and 22Rvl cells were then transduced for 24-48 h with equal amounts of virus followed by selection with media containing blasticidin $(7.5 \mu \mathrm{g} / \mathrm{mL}$ for LNCaP cells, $6 \mu \mathrm{g} / \mathrm{mL}$ for $22 \mathrm{Rv} 1$ cells). Upon selection, clones were derived by serial dilution with subsequent single cell seeding into 96-well plates containing selection media. Cas9 protein expression for each clone was then assessed through Western blotting $\left(1^{\circ} \mathrm{Ms}-\mathrm{Cas} 9\right.$ (Cell Signalling Technology, Cat. No. \#14697) 1:1000, Ms-GAPDH 1:5000 (Santa Cruz Biotechnology, Cat. No. \#sc47724) in 5\% non-fat milk; $2^{\circ}$ HRP-linked Anti-Mouse IgG (Cell Signalling Technology, Cat. No. \#7076 S) 1:10,000 in 2.5\% non-fat milk. The full unprocessed blot is in the Source Data File.

Lentiviral particles were generated in 293FT cells (ThermoFisher) using the pMDG. 2 and psPAX2 packaging plasmids (Addgene; \#12259 and \#12260, a gift from Didier Trono) alongside the Lenti-dCas9-KRAB-blast plasmid (Addgene \#89567, a gift from Gary Hon) and collected $72 \mathrm{hrs}$ post transfection. LNCaP and $22 \mathrm{Rv} 1$ cells were then transduced for $24-48 \mathrm{~h}$ with equal amounts of virus followed by selection with media containing blasticidin $(7.5 \mu \mathrm{g} / \mathrm{mL}$ for LNCaP cells, $6 \mu \mathrm{g} / \mathrm{mL}$ for 22Rv1 cells). Upon selection, clones were derived by serial dilution with subsequent single cell seeding into 96 -well plates containing selection media. dCas9-KRAB protein expression for each clone was then assessed through western blotting ( $1^{\circ}$ Ms-Cas9 (Cell Signalling Technology, Cat. No. \#14697) 1:1000, MsGAPDH 1:5000 (Santa Cruz Biotechnology, Cat. No. \#sc47724) in 5\% non-fat milk; $2^{\circ}$ HRP-linked Anti-Mouse IgG (Cell Signalling Technology, Cat. No. \#7076 S) $1: 10,000$ in $2.5 \%$ non-fat milk. The full unprocessed blot is in the Source Data File.

For gRNA design, five to six unique crRNA molecules (Integrated DNA Technologies) were designed to tile across the region of interest using the CRISPOR tool (http://crispor.tefor.net// ${ }^{67}$ and the Zhang lab CRISPR Design tools (http://crispr.mit.edu/) ${ }^{68}$ (Supplementary Data). Each CRISPR RNA (crRNA) and tracrRNA (Integrated DNA Technologies) were duplexed according to company supplier protocol to a concentration of $50 \mu \mathrm{M}$. Upon generation of the clones, six guides (crRNA-tracrRNA duplexes) for each region of interest were pooled into a single tube $(1 \mu \mathrm{L}$ each guide, $6 \mu \mathrm{L}$ per reaction) (Integrated DNA Technologies).
At last, $1 \mu \mathrm{L}(100 \mu \mathrm{M})$ of electroporation enhancer (Integrated DNA Technologies) was added to the mix $(7 \mu \mathrm{L}$ total) prior to transfection. The entire transfection reaction was transfected into 350,000 cells through Nucleofection (SF Solution EN120-4D Nucleofector, Lonza). Cells were then harvested $24 \mathrm{~h}$ post transfection for RNA and DNA for RT-PCR and confirmation of deletion, respectively.

Transient Cas9-mediated disruption of CREs. Deletion of elements through this method were achieved through the transfection of Cas 9 nuclease protein complexed with the crRNA (Integrated DNA Technologies). In brief, five to six unique crRNA molecules (Integrated DNA Technologies) were designed to tile across the region of interest using the CRISPOR tool (http://crispor.tefor.net/) ${ }^{67}$ and the Zhang lab CRISPR Design tools (http://crispr.mit.edu/) ${ }^{68}$. Each crRNA and tracrRNA (Integrated DNA Technologies) were duplexed according to company supplier protocol to a concentration of $50 \mu \mathrm{M}$. The six crRNA-tracrRNA duplexes were pooled into a single tube $(6 \mu \mathrm{L}$ per reaction), prior to adding $1 \mu \mathrm{L}(5 \mu \mathrm{g})$ of Alt-R S.p HiFi Cas9 Nuclease 3NLS (Integrated DNA Technologies). The reaction was incubated at room temperature for $10 \mathrm{~min}$ for RNP complex formation. At last, $1 \mu \mathrm{L}(100 \mu \mathrm{M})$ of electroporation enhancer (Integrated DNA Technologies) was added to the mix prior to transfection. The entire transfection reaction was transfected into 350,000 cells through Nucleofection (SF Solution EN120-4D Nucleofector, Lonza). Cells were then harvested $24 \mathrm{~h}$ post transfection for RNA and DNA for RT-PCR and confirmation of deletion, respectively. For double deletions, two sets of guide RNA-RNP complex (10 $\mu \mathrm{g}$ of Alt-R S.p HiFi Cas9 Nuclease 3NLS) were transfected and harvested $24 \mathrm{~h}$ post transfection for RNA and DNA for RT PCR and confirmation of deletion, respectively. To control for double deletions, two negative control regions within the TAD were also compounded (Supplementary Data).

RT-PCR assessment of gene expression upon deletion of CREs. DNA and RNA were harvested with Qiagen AllPrep RNA/DNA Kit (Qiagen, Cat. No. 80204). Next, cDNA was synthesized from 300 ng of RNA using SensiFast cDNA Synthesis kit (Bioline, Cat. No. BIO-65054), and mRNA expression levels for various genes of interest were assessed. The primer sequences for expression evaluation are in Supplementary Data. Differential gene expression was calculated upon normalization with TBP (housekeeping gene). Statistical significance was calculated using Student's $t$ test in $\mathrm{R}$.

Confirmation of Cas9-mediated deletion of CREs. Deletion of CREs were confirmed through PCR amplification of the intended region for deletion, followed by the T7 endonuclease assay (Integrated DNA Technology). Primer sequences used for PCR amplification are in Supplementary Data. PCR products were then loaded onto a $1 \%$ agarose gel for visualization. The agarose gel to assess the on-target genome editing efficiency was done through densitometry using ImageJ. The correlation between on-target genome editing efficiency and FOXA1 mRNA expression reduction was drawn through Pearson's correlation in $\mathrm{R}$.

\section{Cell proliferation upon deletion of FOXA1 CREs. Pairs of gRNAs flanking} the CREs of interest, FOXA1 promoter and control regions were designed using CRISPOR (http://crispor.tefor.net/) and Zhang lab CRISPR Design tool (http://crispr.mit.edu/) (Supplementary Data). Each pair of gRNAs were cloned into the lentiCRISPRv2 (Addgene; a gift from Feng Zhang \#52961) and the lentiCRISPRv2-Blast (Addgene; a gift from Feng Zhang \#83480) plasmid as previously described ${ }^{69}$. Lentiviral particles were generated in 293FT cells (ThermoFisher) using the pMDG.2 and psPAX2 packaging plasmids (Addgene; \#12259 and \#12260, a gift from Didier Trono), and collected $72 \mathrm{hrs}$ post transfection. LNCaP cells were transduced for $24-48 \mathrm{hrs}$ with equal amounts of virus, followed by selection with media containing puromycin $(3.5 \mu \mathrm{g} / \mathrm{mL}$, ThermoFisher) and blasticidin $(7 \mu \mathrm{g} / \mathrm{mL}$, Wisent). Cells were harvested upon selection for RNA and DNA for RT-PCR and confirmation of DNA cleavage, respectively. For cell proliferation, cells were seeded at equal density per well (on a 96-well plate; Day 1) upon puromycin and blasticidin selection. Growth of the cells were monitored by cell counting using Countess automated cell counter (Invitrogen). Cell numbers were calculated as a percentage compared to negative control. Statistical significance was calculated using Student's $t$ test.

Luciferase reporter assays. Each region of interest was ordered as gBlocks from Integrated DNA Technologies. The regions were cloned into the BamHI restriction enzyme digest site of the pGL3 promoter plasmid (Promega). On Day 0, 90,000 LNCaP cells were seeded in 24-well plates. Next day (Day 1), pGL3 plasmids harboring the wild-type and variant sequences were co-transfected with the pRL Renilla plasmid (Promega) using Lipofectamine 2000. $48 \mathrm{~h}$ later, the cells were harvested, and dual luciferase reporter assays were conducted (Promega). Notably, inserts of both forward and reverse directions were tested using this assay as enhancer elements are known to be direction-independent. Final luminescence readings are reported as firefly luciferase normalized to renilla luciferase activity. The assessment of each mutation was conducted in five biological replicates. Statistical significance was assessed by Mann-Whitney $U$ test in R. gBlock sequences are in Supplementary Data. 
Allele-specific ChIP-qPCR. In brief, pGL3 plasmids containing the wild-type sequence and the mutant sequence used in the luciferase reporter assay were transfected into 7 million cells $(2 \mu \mathrm{g}$ per allele, per one million cells) using Lipofectamine 2000 (ThermoFisher Scientific), per manufacturer's instructions. Next day, each antibody (FOXA1 $5 \mu \mathrm{g}$, Abcam, ab23738; AR $5 \mu \mathrm{g}$, Abcam, ab1083241; HOXB13 $5 \mu \mathrm{g}$, Abcam, ab201682; SOX9 $5 \mu \mathrm{g}$, Abcam, ab3697; GATA2 $5 \mu \mathrm{g}$, Abcam, ab22849; FOXP1 $5 \mu \mathrm{g}$, Abcam, ab16645; NKX3.1 $10 \mu$ l, Cell Signalling Technology, \#83700) was conjugated with $10 \mu \mathrm{L}$ of each Dynabeads A and G (ThermoFisher Scientific) for each ChIP for $6 \mathrm{~h}$ with rotation at $4{ }^{\circ} \mathrm{C}$. When antibody beads conjugates were ready for use, cells were lifted using trypsin and fixed by resuspending with $300 \mu \mathrm{L}$ of $1 \%$ formaldehyde in PBS for $10 \mathrm{~min}$ at room temperature. $2.5 \mathrm{M}$ Glycine was added to quench excess formaldehyde (final concentration $0.125 \mathrm{M}$ ). Cells were then washed with cold PBS and lysed using $300 \mu \mathrm{L}$ of Modified RIPA buffer (10 mM TrisHCl, pH 8.0; 1 mM EDTA; $140 \mathrm{mM}$ $\mathrm{NaCl} ; 1 \%$ Triton X-100; $0.1 \%$ SDS; $0.1 \%$ sodium deoxycholate) supplemented with protease inhibitor. The lysate was subject to 25 cycles of sonication $(30 \mathrm{~s}$ ON $30 \mathrm{~s}$ OFF) using Diagenode Bioruptor Pico (Diagenode). In all, $15 \mu \mathrm{L}$ of sonicated lysate was set aside as input with the rest used for chromatin pulldown through addition of antibody beads conjugates and overnight incubation at $4{ }^{\circ} \mathrm{C}$ with rotation. Next day, the beads were washed once with Modified RIPA buffer, washed once with Modified RIPA buffer $+500 \mathrm{mM} \mathrm{NaCl}$, once with LiCl buffer $(10 \mathrm{mM}$ TrisHCl, pH 8.0; $1 \mathrm{mM}$ EDTA; $250 \mathrm{mM} \mathrm{LiCl} ; 0.5 \% \mathrm{NP}-40 ; 0.5 \%$ sodium deoxycholate) and twice with Tris-ETDA buffer ( $\mathrm{pH} 8$ ). After wash, beads and input were decrosslinked by addition of $100 \mu \mathrm{L}$ Decrosslinking buffer and incubation at $65^{\circ} \mathrm{C}$ for $6 \mathrm{~h}$. Samples were then purified and eluted. ChIP and input DNA were then used for allele-specific ChIP-qPCR using MAMA primers as described previously (Supplementary Data). Fold-change significance was calculated using Student's $t$ test in R.

All analyses were done using hg19 reference genome coordinates.

Reporting summary. Further information on research design is available in the Nature Research Reporting Summary linked to this article.

\section{Data availability}

Genomic and Epigenomic data sets used to support this study can be found from the following accession codes: primary tumors-H3K27ac ChIP-seq (GSE96652), SNVs called from primary tumors (https://dcc.icgc.org/projects/PRAD-CA), FOXA1, AR, and HOXB13 ChIP-seq in primary prostate tumors is available under the following accession code: GSE137527 and EGAS00001003928, TF ChIP-seq data were from public databases of ReMap and ChIP-Atlas. All other relevant data supporting the key findings of this study are available within the article and its Supplementary Information files or from the corresponding author upon reasonable request. Data used to generate the figures are available in the Source Data file. A reporting summary for this Article is available as a Supplementary Information file.

Received: 27 January 2019; Accepted: 20 December 2019; Published online: 23 January 2020

\section{References}

1. Bray, F. et al. Global cancer statistics 2018: GLOBOCAN estimates of incidence and mortality worldwide for 36 cancers in 185 countries. CA Cancer J. Clin. 68, 394-424 (2018).

2. Boorjian, S. A. et al. Long-term outcome after radical prostatectomy for patients with lymph node positive prostate cancer in the prostate specific antigen era. J. Urol. 178, 864-870 (2007).

3. Litwin, M. S. \& Tan, H.-J. The diagnosis and treatment of postate cancer: a review. JAMA 317, 2532-2542 (2017).

4. Attard, G. et al. Prostate cancer. Lancet 387, 70-82 (2016).

5. Cancer Genome Atlas Research Network. The molecular taxonomy of primary prostate cancer. Cell 163, 1011-1025 (2015).

6. Fraser, M. et al. Genomic hallmarks of localized, non-indolent prostate cancer. Nature 541, 359-364 (2017).

7. Barbieri, C. E. et al. Exome sequencing identifies recurrent SPOP, FOXA1 and MED12 mutations in prostate cancer. Nat. Genet. 44, 685-689 (2012).

8. Grasso, C. S. et al. The mutational landscape of lethal castration-resistant prostate cancer. Nature 487, 239-243 (2012).

9. Parolia, A. et al. Distinct structural classes of activating FOXA1 alterations in advanced prostate cancer. Nature 571, 413-418 (2019).

10. Adams, E. J. et al. FOXA1 mutations alter pioneering activity, differentiation and prostate cancer phenotypes. Nature 571, 408-412 (2019).

11. Robinson, D. et al. Integrative clinical genomics of advanced prostate cancer. Cell 161, 1215-1228 (2015).

12. Robinson, J. L. L., Holmes, K. A. \& Carroll, J. S. FOXA1 mutations in hormone-dependent cancers. Front. Oncol. 3, 20 (2013).
13. Gao, S. et al. Forkhead domain mutations in FOXA1 drive prostate cancer progression. Cell Res. 29, 770-772 (2019)

14. Annala, M. et al. Frequent mutation of the FOXA1 untranslated region in prostate cancer. Commun. Biol. 1, 122 (2018).

15. Yang, Y. A. \& Yu, J. Current perspectives on FOXA1 regulation of androgen receptor signaling and prostate cancer. Genes Dis. 2, 144-151 (2015).

16. Lupien, $M$. et al. FoxA1 translates epigenetic signatures into enhancer-driven lineage-specific transcription. Cell 132, 958-970 (2008).

17. Eeckhoute, J. et al. Cell-type selective chromatin remodeling defines the active subset of FOXA1-bound enhancers. Genome Res. 19, 372-380 (2009).

18. Pomerantz, M. M. et al. The androgen receptor cistrome is extensively reprogrammed in human prostate tumorigenesis. Nat. Genet. 47, 1346-1351 (2015).

19. Imamura, Y. et al. FOXA1 promotes tumor progression in prostate cancer via the insulin-like growth factor binding protein 3 pathway. PLoS ONE 7, e42456 (2012).

20. Jin, H.-J., Zhao, J. C., Ogden, I., Bergan, R. C. \& Yu, J. Androgen receptorindependent function of FoxA1 in prostate cancer metastasis. Cancer Res. 73, 3725-3736 (2013).

21. Xu, Y., Chen, S.-Y., Ross, K. N. \& Balk, S. P. Androgens induce prostate cancer cell proliferation through mammalian target of rapamycin activation and post-transcriptional increases in cyclin D proteins. Cancer Res. 66, 7783-7792 (2006).

22. Yang, Y. A. et al. FOXA1 potentiates lineage-specific enhancer activation through modulating TET1 expression and function. Nucleic Acids Res. 44, 8153-8164 (2016).

23. Zhang, G. et al. FOXA1 defines cancer cell specificity. Sci. Adv. 2, e1501473 (2016).

24. Augello, M. A., Hickey, T. E. \& Knudsen, K. E. FOXA1: master of steroid receptor function in cancer. EMBO J. 30, 3885-3894 (2011).

25. Sunkel, B. et al. Integrative analysis identifies targetable CREB1/FoxA1 transcriptional co-regulation as a predictor of prostate cancer recurrence. Nucleic Acids Res. 45, 6993 (2017).

26. Ni, M. et al. Amplitude modulation of androgen signaling by c-MYC. Genes Dev. 27, 734-748 (2013).

27. Sasse, S. K. \& Gerber, A. N. Feed-forward transcriptional programming by nuclear receptors: regulatory principles and therapeutic implications. Pharmacol. Ther. 145, 85-91 (2015).

28. Wang, S., Singh, S. K., Katika, M. R., Lopez-Aviles, S. \& Hurtado, A. High throughput chemical screening reveals multiple regulatory proteins on FOXA1 in breast cancer cell lines. Int. J. Mol. Sci. 19, pii: E4123 (2018).

29. Rowley, M. J. \& Corces, V. G. Organizational principles of 3D genome architecture. Nat. Rev. Genet. 19, 789-800 (2018).

30. Vernimmen, D. \& Bickmore, W. A. The hierarchy of transcriptional activation: from enhancer to promoter. Trends Genet. 31, 696-708 (2015).

31. Sallari, R. C. et al. Convergence of dispersed regulatory mutations predicts driver genes in prostate cancer. (2017) https://doi.org/10.1101/097451.

32. Bailey, S. D. et al. Noncoding somatic and inherited single-nucleotide variants converge to promote ESR1 expression in breast cancer. Nat. Genet. (2016) https://doi.org/10.1038/ng.3650.

33. Tsourlakis, M. C. et al. FOXA1 expression is a strong independent predictor of early PSA recurrence in ERG negative prostate cancers treated by radical prostatectomy. Carcinogenesis 38, 1180-1187 (2017).

34. Phillips, J. E. \& Corces, V. G. CTCF: master weaver of the genome. Cell 137 1194-1211 (2009).

35. Weintraub, A. S. et al. YY1 is a structural regulator of enhancer-promoter loops. Cell 171, 1573-1588.e28 (2017).

36. Bailey, S. D. et al. ZNF143 provides sequence specificity to secure chromatin interactions at gene promoters. Nat. Commun. 2, 6186 (2015).

37. Mehdi, T., Bailey, S. D., Guilhamon, P., Lupien, M. \& Kelso, J. C3D: a tool to predict 3D genomic interactions between cis-regulatory elements. Bioinformatics (2018) https://doi.org/10.1093/bioinformatics/bty717.

38. Kron, K. J. et al. TMPRSS2-ERG fusion co-opts master transcription factors and activates NOTCH signaling in primary prostate cancer. Nat. Genet. (2017) https://doi.org/10.1038/ng.3930.

39. Creyghton, M. P. et al. Histone H3K27ac separates active from poised enhancers and predicts developmental state. Proc. Natl Acad. Sci. USA 107, 21931-21936 (2010).

40. Espiritu, S. M. G. et al. The evolutionary landscape of localized prostate cancers drives clinical aggression. Cell 173, 1003-1013.e15 (2018).

41. DeKelver, R. C. et al. Functional genomics, proteomics, and regulatory DNA analysis in isogenic settings using zinc finger nuclease-driven transgenesis into a safe harbor locus in the human genome. Genome Res. 20, 1133-1142 (2010).

42. Pennacchio, L. A., Bickmore, W., Dean, A., Nobrega, M. A. \& Bejerano, G. Enhancers: five essential questions. Nat. Rev. Genet. 14, 288-295 (2013).

43. Rheinbay, E. et al. Recurrent and functional regulatory mutations in breast cancer. Nature 547, 55-60 (2017). 
44. Zhang, $\mathrm{X}$. et al. Integrative functional genomics identifies an enhancer looping to the SOX9 gene disrupted by the $17 \mathrm{q} 24$. 3 prostate cancer risk locus. Genome Res. 22, 1437-1446 (2012).

45. Huang, F. W. et al. Highly recurrent TERT promoter mutations in human melanoma. Science 339, 957-959 (2013).

46. Horn, S. et al. TERT promoter mutations in familial and sporadic melanoma. Science 339, 959-961 (2013).

47. Fuxman Bass, J. I. et al. Human gene-centered transcription factor networks for enhancers and disease variants. Cell 161, 661-673 (2015).

48. Zhou, S., Treloar, A. E. \& Lupien, M. Emergence of the noncoding cancer genome: a target of genetic and epigenetic alterations. Cancer Discov. 6, 1215-1229 (2016).

49. Feigin, M. E. et al. Recurrent noncoding regulatory mutations in pancreatic ductal adenocarcinoma. Nat. Genet. 49, 825-833 (2017).

50. Khurana, E. et al. Role of non-coding sequence variants in cancer. Nat. Rev. Genet. 17, 93-108 (2016)

51. Cowper-Sal, lari,R. et al. Breast cancer risk-associated SNPs modulate the affinity of chromatin for FOXA1 and alter gene expression. Nat. Genet. 44, 1191-1198 (2012).

52. Rhie, S. K. et al. A high-resolution $3 \mathrm{D}$ epigenomic map reveals insights into the creation of the prostate cancer transcriptome. Nat. Commun. 10, 4154 (2019).

53. Liu, Q. et al. Disruption of a $-35 \mathrm{~kb}$ enhancer impairs CTCF binding and MLH1 expression in colorectal cells. Clin. Cancer Res. (2018) https://doi.org/ 10.1158/1078-0432.CCR-17-3678.

54. Zhang, X. et al. Identification of focally amplified lineage-specific superenhancers in human epithelial cancers. Nat. Genet. 48, 176-182 (2016).

55. Takeda, D. Y. et al. A somatically acquired enhancer of the androgen receptor is a noncoding driver in advanced prostate cancer. Cell (2018). https://doi.org/ 10.1016/j.cell.2018.05.037.

56. Viswanathan, S. R. et al. Structural alterations driving castration-resistant prostate cancer revealed by linked-read genome sequencing. Cell (2018) https://doi.org/10.1016/j.cell.2018.05.036.

57. Osterwalder, M. et al. Enhancer redundancy provides phenotypic robustness in mammalian development. Nature 554, 239-243 (2018).

58. Melton, C., Reuter, J. A., Spacek, D. V. \& Snyder, M. Recurrent somatic mutations in regulatory regions of human cancer genomes. Nat. Genet. 47, 710-716 (2015).

59. Mazrooei, P. et al. Cistrome Partitioning Reveals Convergence of Somatic Mutations and Risk Variants on Master Transcription Regulators in Primary Prostate Tumors. (2019) https://doi.org/10.1016/j.ccell.2019.10.005.

60. Weinhold, N., Jacobsen, A., Schultz, N., Sander, C. \& Lee, W. Genome-wide analysis of noncoding regulatory mutations in cancer. Nat. Genet. $\mathbf{4 6}$, 1160-1165 (2014).

61. Wedge, D. C. et al. Sequencing of prostate cancers identifies new cancer genes, routes of progression and drug targets. Nat. Genet. 50, 682-692 (2018).

62. Quigley, D. A. et al. Genomic hallmarks and structural variation in metastatic prostate cancer. Cell (2018) https://doi.org/10.1016/j.cell.2018.06.039.

63. Cancer Cell Line Encyclopedia Consortium \& Genomics of Drug Sensitivity in Cancer Consortium. Pharmacogenomic agreement between two cancer cell line data sets. Nature 528, 84-87 (2015).

64. McFarland, J. M. et al. Improved estimation of cancer dependencies from large-scale RNAi screens using model-based normalization and data integration. Nat. Commun. 9, 4610 (2018).

65. Thurman, R. E. et al. The accessible chromatin landscape of the human genome. Nature 489, 75-82 (2012).

66. Wang, Y. et al. The 3D Genome Browser: a web-based browser for visualizing 3D genome organization and long-range chromatin interactions. bioRxiv 112268 (2017) https://doi.org/10.1101/112268.

67. Haeussler, M. et al. Evaluation of off-target and on-target scoring algorithms and integration into the guide RNA selection tool CRISPOR. Genome Biol. 17, 148 (2016)

68. Hsu, P. D. et al. DNA targeting specificity of RNA-guided Cas9 nucleases. Nat. Biotechnol. 31, 827-832 (2013).

69. Sanjana, N. E., Shalem, O. \& Zhang, F. Improved vectors and genome-wide libraries for CRISPR screening. Nat. Methods 11, 783-784 (2014).

\section{Acknowledgements}

We thank the Princess Margaret Genomics Centre and the Princess Margaret Bioinformatics group for providing support and infrastructure for the computational analysis of this work as well as high-throughput sequencing support and the NKI Core Facility Molecular Pathology and Biobanking for technical support and providing tissue specimens. We thank all the members of the M.L. lab for their fruitful discussions and feedback. This work is supported Prostate Cancer Canada, Ontario Institute for Cancer Research funded by the Government of Ontario, The Princess Margaret Cancer Foundation (M.L. and R.G.B.), Movember Foundation (RS2014-04 to M.L. and RS2014-01 to P.C.B.), the Radiation Medicine Program Academic Enrichment Fund (R.G.B.), Terry Fox Research Institute New Investigator Award (P.C.B.), Canadian Institute of Health Research (CIHR) (FRN-153234 to M.L.), and New Investigator Award (P.C.B. and M.L.), Canadian Cancer Society Research Scientist Award (R.G.B.), Cancer Cancer Society Impact Award (P.C.B), the Dutch Cancer Society KWF/Alpe d'HuZes (10084 and NKI 2014-6711 ALPE to W.Z.), CIHR Graduate Scholarship-Master's Research Award (J.R. H., M.T.). CIHR Graduate Scholarship-Doctoral Research Award (P.M., J.T.H.), Canadian Breast Cancer Foundation (CBCF) postdoctoral fellowship (K.J.K.), Investigator Award from the Ontario Institute for Cancer Research (M.L. and P.C.B.) and Movember Rising Star Award from Prostate Cancer Canada (M.L. and P.C.B.).

\section{Author contributions}

S.Z. and M.L. conceptualized the study. S.Z. designed and conducted most of the experiments with help from F.S., G.G., M.T., K.J.K., J.T.H., C.A., H.Y.Y., Y.Z. and S.C J.R.H. implemented most of the computational analyses and statistical approaches with help from S.A.M., P.M., M.A., A.M., V.H., T.N.Y., S.M.G.E., T.M.S. and J.L. under the supervision of W.Z., T.v.d.K., T.J.P., M.F., P.C.B., R.G.B., H.H.H., or M.L. Figures were designed by S.Z. with assistance from J.R.H. and S.A.M. The manuscript was written by S.Z., J.R.H. and M.L. with assistance from all authors. M.L. oversaw the study.

\section{Competing interests}

W.Z. receives project funding from Astellas Pharma but the components of this manuscript provided by W.Z. and his team were funded by KWF Dutch Cancer Society and Oncode Institute. The other authors declare no competing interests.

\section{Additional information}

Supplementary information is available for this paper at https://doi.org/10.1038/s41467020-14318-9.

Correspondence and requests for materials should be addressed to M.L.

Peer review information Nature Communications thanks the anonymous reviewer(s) for their contribution to the peer review of this work. Peer reviewer reports are available.

Reprints and permission information is available at http://www.nature.com/reprints

Publisher's note Springer Nature remains neutral with regard to jurisdictional claims in published maps and institutional affiliations.

\footnotetext{
Open Access This article is licensed under a Creative Commons Ay Attribution 4.0 International License, which permits use, sharing,
adaptation, distribution and reproduction in any medium or format, as long as you give appropriate credit to the original author(s) and the source, provide a link to the Creative Commons license, and indicate if changes were made. The images or other third party material in this article are included in the article's Creative Commons license, unless indicated otherwise in a credit line to the material. If material is not included in the article's Creative Commons license and your intended use is not permitted by statutory regulation or exceeds the permitted use, you will need to obtain permission directly from the copyright holder. To view a copy of this license, visit http://creativecommons.org/ licenses/by/4.0/.
}

(C) The Author(s) 2020 